\title{
Antimicrobial, antioxidant, antitumour-promoting and cytotoxic activities of different plant part extracts of Garcinia atroviridis Griff. ex T. Anders
}

\begin{abstract}
Crude extracts (methanol) of various parts, viz. the leaves, fruits, roots, stem and trunk bark, of Garcinia atroviridis were screened for antimicrobial, cytotoxic, brine shrimp toxic, antitumour-promoting and antioxidant activities. The crude extracts exhibited predominantly antibacterial activity with the root extract showing the strongest inhibition against the test bacteria at a minimum inhibitory dose (MID) of $15.6 \mathrm{gg} / \mathrm{disc}$. Although all the extracts failed to inhibit the growth of most of the test fungi, significant antifungal activity against Cladosporium herbarum was exhibited by most notably the fruit (MID: $100 \mathrm{gg}$ ), and the leaf (MID: $400 \varepsilon \mathrm{g}$ ) extracts. None of the extracts were significantly cytotoxic, and lethal towards brine shrimps. The root, leaf, trunk and stem bark extracts (except for the fruits) showed strong antioxidant activity exceeding that of the standard antioxidant, Ú-tocopherol. Antitumour-promoting activity (>95\% inhibition) was shown by the fruit, leaf, stem and trunk bark extracts.
\end{abstract}

Keyword: Garcinia atroviridis; Antimicrobial; Antioxidant; Antitumour-promoting 